\title{
Learning from failings in healthcare: a challenge for all healthcare systems
}

\author{
Fiona Moss
}

\section{Might Mid Staffs be a turning point?}

\begin{abstract}
The most important single change in the NHS in response to this report would be for it to become more than ever before a system devoted to continual learning and improvement of patient care, top to bottom, end to end. ${ }^{1}$
\end{abstract}

Reactions to significant and public failures in healthcare in the UK, and no doubt elsewhere in the world, trigger forensic inquiries. The aim, after finding out what went wrong, is to draw out lessons with the aim of ' it' never happening again. Each inquiry, whether small and internal or large and statutory, publishes a report with recommendations. These reports make difficult reading for anyone working in healthcare. For although the context and clinical details are never quite the same, they point to similar and familiar failures. The latest public failing in the UK has triggered not one but two inquiries and three reports: a report followed each inquiry with a third from an expert group, chaired by Professor Don Berwick, charged with taking the lessons from this latest failing and specifying what changes are needed to make the National Health Service (NHS) a safer health system. Will this third report, written to, and for, everyone in the NHS, make a lasting difference?

The context for the Berwick report is the care provided at Mid Staffordshire Trust, a district hospital in the English West Midlands. Concerns about standards and the safety of care, and a higher than expected death rate, alerted the regulator, the Health Care Commission, which in 2009 investigated this hospital and published a report very critical of standards of care. There followed reports by the Department of Health and, later in 2009, the Secretary of State for Health commissioned an independent inquiry chaired by Robert Francis QC, which reported in $2010 .^{2}$ But continued widespread public disquiet and anger, reflected through pressure groups and media coverage, about the extent of harm to patients at this hospital

Correspondence to Dr Fiona Moss, Editor in Chief, Postgraduate Medical Journal, BMJ, BMA House, Tavistock Square, London WC1H 9JR, UK; pmj@bmj.com prompted a public independent inquiry also chaired by Robert Francis. Building on the first inquiry, the second looked beyond the hospital to the wider healthcare system and considered, for example, the effectiveness of the supervisory and regulatory organisations. The objectives of this second report included identifying lessons that would enable the NHS to recognise failing hospitals sooner. The final report published in 2013-Analysis of Evidence and Lessons Learned-has 290 recommendations that reach into most parts of the NHS. $^{3}$

Both Francis reports reflect a tragedy affecting many people played out slowly over several years-replays have since provoked a background anxiety in the NHS. Some patients died when they should have lived; many more were harmed or received appalling care; and many patients and their relatives suffered. The inquiry concluded that the failings started at the top with a board which had somehow allowed a negative culture, tolerant of poor care, to pervade the hospital, and it is clear that many of the 'checks and balances' that should have prevented failings (including organisations with oversight of the hospital) simply did not work. ${ }^{3}$ The temptation in the face of such failings is to seek whom to blame. But the inquiry is clear: this was essentially a system failure. The government, determined that there will not be another 'Mid Staffs', responded robustly and has, for example, introduced a chief inspector of hospitals and a new rating system for hospitals.

For Francis the key is in the culture of a hospital. Patients looked after by people working in a culture of openness and honesty that fosters good, wellfunctioning teams will receive much better and safer care than people, such as many of those cared for at Mid Staffordshire, whose treatment depends on staff who are disengaged and working in an atmosphere of fear and isolation. Changing the culture of any organisation is tough. New and ingenious strategies and well worked through project plans have their place but they are unlikely to make an impact on the culture of an organisation: 'culture eats strategy for breakfast'. ${ }^{4}$ Pressuring people to 'do better' or promoting change through fear or regulation at best will produce workarounds or gaming and is very unlikely to result in lasting commitment to change.

Just publishing the findings of inquiries seems unlikely to make a difference: we have four decades of experience of the apparent lack of impact of such reports. In 2002 Walshe and Higgins compared the reports of over 50 major inquiries into failures in the NHS from 1972 to 2001 and found that "Many inquiry reports highlight similar sorts of failures, suggesting that lessons are not always learnt. Often these failures are organisational and cultural, and the necessary changes are not likely to happen simply because they are prescribed in a report". 5 They identified five common themes (box 1).

This time, in a change from usual practice, while public and media interest remained fresh and raw and those in the health service were still sensitised to the possibility that there might be 'other Mid-Staffs', the government asked Professor Don Berwick to chair a National Advisory Group on the Safety of Patients in England and to set out recommendations and specify the changes needed for the NHS to deliver consistently better and safer care. Free from the need to collect and collate evidence from a specific place and event, the Berwick Committee, made up of academics and experts in organisational theory, quality improvement, safety and systems, used the tragedy of 'Mid Staffs' and Francis'

\section{Box 1 Common themes of inquiries ${ }^{5}$}

- Organisational or geographical isolation-which inhibits the transfer of innovation and hinders peer review and constructive critical exchange.

- Inadequate leadership_lacking vision and unwilling to tackle known problems.

- System and process failure-in which organisational systems and processes are either absent or not working properly.

- Poor communication - both within the NHS organisation and between it and patients or clients, which means that problems are not picked up.

- Disempowerment of staff and patients - which means that those who might have raised concerns were discouraged or prevented from doing so. 
Box 3 Berwick Committee's four principles for the NHS as a learning organisation $^{1}$

The NHS should continually and forever reduce patient harm by embracing wholeheartedly an ethic of learning.

- All leaders concerned with NHS healthcare-political, regulatory, governance, executive, clinical and advocacy - should place quality of care in general, and patient safety in particular, at the top of their priorities for investment, inquiry, improvement, regular reporting, encouragement and support.

- Patients and their carers should be present, powerful and involved at all levels of healthcare organisations from wards to the boards of trusts.

- Government, Health Education England and NHS England should ensure that sufficient staff are available to meet the NHS's needs now and in the future. Healthcare organisations should ensure that staff are present in appropriate numbers to provide safe care at all times and are well supported.

- Mastery of quality and patient safety sciences and practices should be part of initial preparation and lifelong education of all healthcare professionals, including managers and executives.

- The NHS should become a learning organisation. Its leaders should create and support the capability for learning, and therefore change, at scale, within the NHS

- Transparency should be complete, timely and unequivocal. All data on quality and safety, whether assembled by government, organisations, or professional societies, should be shared in a timely fashion with all parties who want it, including, in accessible form, with the public.

- All organisations should seek out the patient and carer voice as an essential asset in monitoring the safety and quality of care.

- Supervisory and regulatory systems should be simple and clear. They should avoid diffusion of responsibility. They should be respectful of the goodwill and sound intention of the vast majority of staff. All incentives should point in the same direction.

- We should support responsive regulation of organisations, with a hierarchy of responses. Recourse to criminal sanctions should be extremely rare, and should function primarily as a deterrent to wilful or reckless neglect or mistreatment.

detailed reports on its failings as a starting point for its analysis.

The result is a report with a difference. Written simply, straightforwardly and concisely it outlines 10 recommendations and includes four main principles to guide everyone trying to build an even better 'learning NHS' (boxes 2 and 3). In a letter to the people of England, it outlines the role of patients and a set of actions for patients and carers. Reaching out in another letter to all 'clinicians, managers and staff in the NHS' this report acknowledges their impressive work and early on states clearly that 'NHS staff are not to blame'. But although understanding, this report is challenging and uncompromising. 'The only conceivably worthy honour due to those harmed', it begins 'is to make changes that will save other people and other places from similar harm'.

Learning is a key underlying theme. Continual improvement and safer systems are unlikely to spring from inspections or from external mandates. The way to a better, safer NHS lies with those who work in it; but this will require putting into action the recommendations of the Berwick report. Currently, within the NHS, learning and education and training focuses mostly on those in formal training programmes. Continual professional and personal development (CPPD) does happen but largely at an individual level and only for some staff. The point is that learning must become integral to the working lives and working practices of all NHS staff-clinical professionals at all stages and managers and support staff alike. Learning should be team based and patient focused. In an environment where teams ask each day 'How have we done?' and 'How could we have done better?' there should be no need for anyone to become a whistleblower. to the development of clinical professionals. But viewed in the light of both the Francis and Berwick reports they seem very narrow. Medical and most other clinical professional training focuses on the treatment and clinical management of individual patients, with little emphasis on organisational skills or learning about
Formal training programmes are central
Place the quality of patient care, especially patient safety, above all other aims.

- Engage, empower and hear patients and carers at all times.

- Foster whole-heartedly the growth and development of all staff, including their ability and support to improve the processes in the area in which they work.

- Embrace transparency unequivocally and everywhere, in the service of accountability, trust, and the growth of knowledge.

organisational behaviour or the skills needed for quality improvement. Of course, the curriculums of disease, diagnosis and therapeutics and the development of specialist skills are hugely important: without them there is no medicine. But we have an approach to education perfectly designed to develop and train professionals who are good at looking after individuals but nowhere near as skilled in looking after the system in which those patients are investigated, treated and cared for.

Looking after patients well requires an understanding both of the needs of individual patients and an understanding of the system of care in order to make it work well for those patients. Focusing only on the individual and without any regard for the system of care will, in the end, harm the individual patient. This is what might have happened at Mid Staffs. So intent on themselves and their patients, it is possible that doctors forgot that patients also need all those looking after them to have good and functional relationships with all their colleagues, clinical and managerial, both within and beyond the hospital, or else there simply isn't a functional system of care: just a series of 'black holes' or a system perfectly designed to produce unsafe care and a culture with the potential to foster unkindnesses.

The skills needed for quality improvement have been written about over the past 20 years, ${ }^{6} 7$ and some will say that current clinical curriculums include some of these skills. But this is to miss the point. What is needed is so much more than 'added extras' in a curriculum: rather 
it is to value team learning as much as the individual pursuit of knowledge and skills. Responsibility for both individual patients and for making the system of care work for patients lies with everyone who works in healthcare. A key leadership task is to make sure that this balance is right. One of the difficulties in putting the Berwick recommendations into action is that many of today's dedicated senior practitioners and those responsible for training curriculums, who have massive influence on how we work and learn, might not have had the opportunity to train in what the Berwick report describes as 'Mastery of quality and safety sciences and practices'. ${ }^{1}$ Those now in training may have a better understanding of what is needed. ${ }^{8}{ }^{9}$ Implicitly, the Berwick report challenges traditional hierarchies: collaboration through networks is what is needed to make a real difference and to ensure that the NHS is a learning organisation and 'safe, effective and patient centred'. Functional teams who work well together, and learn together, will be able collaborate and work with other effective teams to form networks to become an ever evolving and improving system of care. ${ }^{10} 11$

In the end most improvements in care require organisational changes, which means changing the way in which we work together. This is not easily achieved within rigid hierarchies. The daily question for each team: 'How can we do better?' translates into 'Is there a better way of working together that will improve how we deliver care for our patients?'. This can only be achieved if we are aware of, and take responsibility for, how we work togetherand these responsibilities lie most heavily on those who have senior positions in the health system. The Berwick Committee writes, "Make sure that pride and joy in work, not fear, infuse the work of the NHS". "The people who work in health systems matter and they must be cared for so that they can care properly for their patients. Without this how can the NHS ensure both that the core things- the right treatment for the right patient at the right time-happen and that the organisational behaviours that bind these essentials together and attention to important personal details of care are also not neglected?

Most reports into failings end up as failures. There is no magic bullet that will improve care, and 'shouting' at staff through such reports does not work. But by emphasising the importance of learning the Berwick report just might help the NHS become safer. An urgent task for all leaders within the NHS is to ensure that learning becomes part of the fabric of the whole system-'top to bottom, end to end': just what needs to be done is all set out in the Berwick report. Teams whose leaders understand the task, can start this work today: there will be no starting gun. Ensuring good quality and safe care in the end is down to them and how they learn and support their patients and each other, and not to the secretary of state for health and his or her directives. And though written for the NHS, the Berwick report's uncompromising challenges are relevant to all healthcare systems.

\section{Competing interests None.}

Provenance and peer review Commissioned; internally peer reviewed.
To cite Moss F. Postgrad Med J 2013;89:551-553.

Received 29 August 2013

Accepted 29 August 2013

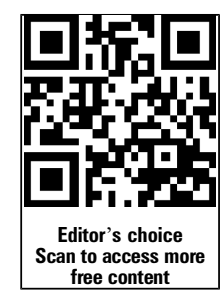

Postgrad Med J 2013;89:551-553.

doi:10.1136/postgradmedj-2013-132345

\section{REFERENCES}

1 Berwick D. National Advisory Group on the Safety of Patients in England. A promise to learn-a commitment to act. 2013. http://www.gov.uk/government/publications/ berwick-review-into-patient-safety Department of Health.

2 Francis R. Independent Inquiry into care provided by Mid Staffordshire NHS Foundation Trust January 2005-March 2009. The Stationary Office, 2010. http://webarchive.nationalarchives.gov.uk/ 20130107105354/http://www.dh.gov.uk/prod consum_dh/groups/dh_digitalassets/@dh/@en/@ps/ documents/digitalasset/dh_113068.pdf

3 Francis R. The mid staffordshire NHS foundation trust public inquiry. London: The Stationary Office, 2013. http://www.midstaffsinquiry.com/

4 Drucker P. http://www.reply-mc.com/people/peter-drucker/

5 Walshe K, Higgins J. The use and impact of inquiries in the NHS. BMJ 2002;325:895-900.

6 Berwick DM, Enthoven A, Bunker JP. Quality management in the NHS: the doctor's role-I. BMJ 1992;304:235-9.

7 Berwick DM, Enthoven A, Bunker JP. Quality management in the NHS: the doctor's role-Il. BMJ 1992;304:304-7.

8 Hillman T, Rouche A. Quality improvement. BMJ careers. April 2011. http://careers.bmj.com/careers/ advice/view-article.html?id=20002524.

9 Lemer C, Moss F. Patient safety and junior doctors: are we missing the obvious? BMJ Qual Saf 2013;22:8-10.

10 Katzenbach JR. Look Beyond the Team: It's About the Network. HBR Blog network. 2012. http://blogs.hbr.org/ cs/2012/03/look beyond the team its about.html

11 Hillman T. Networking in Medicine-an essential clinical skill. 2012 tobyhillman.wordpress.com/2012/ 03/21networking-in-medicine. 